\title{
BMJ Open Access and quality of maternity care for disabled women during pregnancy, birth and the postnatal period in England: data from a national survey
}

Reem Malouf, Jane Henderson, Maggie Redshaw

To cite: Malouf R, Henderson J, Redshaw M. Access and quality of maternity care for disabled women during pregnancy, birth and the postnatal period in England: data from a national survey. BMJ Open 2017;7:e016757. doi:10.1136/ bmjopen-2017-016757

- Prepublication history for this paper is available online. To view these files please visit the journal online (http://dx.doi. org/10.1136/bmjopen-2017016757).

Received 8 March 2017

Revised 17 April 2017

Accepted 5 May 2017

\section{CrossMark}

Policy Research Unit in Maternal Health and Care, National Perinatal Epidemiology Unit, University of Oxford, Oxford, UK

\section{Correspondence to}

Dr Maggie Redshaw;

maggie.redshaw@npeu.ox. ac.uk

\section{ABSTRACT}

Objectives More disabled women are becoming mothers, and yet, their care is rarely the focus of quantitative research. This study aimed to investigate access and quality of maternity care for women with differing disabilities.

Design Secondary analysis was conducted on data from a 2015 national survey of women's experience of maternity care. Descriptive and adjusted analyses were undertaken for five disability groups: physical disability, sensory impairment, mental health disability, learning disability and multiple disability, and comparisons were made with the responses of non-disabled women.

Setting Survey data were collected on women's experience of primary and secondary care in all trusts providing maternity care in England.

Participants Women who had given birth three months previously, among whom were groups self-identifying with different types of disability. Exclusions were limited to women whose baby had died and those who were younger than 16 years at the time of the recent birth.

Results Overall, 20,094 women completed and returned the survey; 1958 women (9.5\%) self-identified as having a disability. The findings indicate some gaps in maternity care provision for these women relating to interpersonal aspects of care: communication, feeling listened to and supported, involvement in decision making, having a trusted and respected relationship with clinical staff. Women from all disability groups wanted more postnatal contacts and help with infant feeding.

Conclusion While access to care was generally satisfactory for disabled women, women's emotional well-being and support during pregnancy and beyond is an area that is in need of improvement. Specific areas identified included disseminating information effectively, ensuring appropriate communication and understanding, and supporting women's sense of control to build trusting relationships with healthcare providers.

\section{BACKGROUND}

The number of disabled women choosing to become mothers is growing. ${ }^{1}$ However, stigma still exists about such women and their care-giving and mothering capabilities. ${ }^{2}$ Although all women are entitled to have
Strengths and limitations of this study

- All organisations providing maternity care in England participated in the recent survey.

- The large size of the survey allowed for more detailed subdivisions and comparison of the experience of different disability groups than previous research.

- Data in this survey were self-reported and collected retrospectively at 3 months postpartum which may affect the quality of responses based on recall.

- The response rate was lower than previous surveys which may affect the generalisability of the findings; however, weighting for non-response was used.

access to high-quality maternity care, worldwide, half of disabled people cannot afford healthcare, compared with a third of non-disabled people, and they are more likely to find healthcare providers' skills inadequate. ${ }^{3}$ This is despite disabled women's greater need for, and use of, healthcare services. ${ }^{4}$ Disabled people and their families frequently experience inequalities in accessing health services, with poor communication and challenging attitudes among healthcare providers. ${ }^{2}$ Furthermore, disabled people are four times more likely to report being treated badly and nearly three times more likely to be denied access to healthcare. ${ }^{3}$

Disabled women accessing maternity care may be considered unusual and problematic. Healthcare professionals may be concerned that these women will not be able to cope with pregnancy and motherhood. ${ }^{5}$ However, the social model of disability suggests that disability is a social construction brought about by structural and attitudinal barriers encountered by people with impairments. ${ }^{6}$ It views disabled people as socially oppressed and argues for policies and practices that facilitate full inclusion. ${ }^{7}$

Healthcare professionals may lack knowledge and experience in planning and 
providing care for pregnant disabled women. ${ }^{8}$ For example, antenatal information may be distributed in a manner inappropriate and insufficient for women with visual impairment. ${ }^{19}$ There is some evidence that women with hearing impairment receive fewer antenatal visits and have limited access to maternity information. ${ }^{1011}$ For women with a less easily identified disability, such as those arising from mental health problems, there may be difficulties in receiving appropriate care. ${ }^{12}$ For women in this group, dissatisfaction and lack of trust have been found to be the main barriers in seeking help during pregnancy. ${ }^{12}$

In the UK, maternity services are freely available for all women. A study reporting on the use of maternity services by women with disabilities in $2010^{13}$ concluded that disabled women were at higher risk for adverse pregnancy outcomes; for example, they were more likely to deliver early and have low-birth-weight babies. However, it also concluded that some women, such as those with physical disabilities, appropriately received more care. In this paper, we aim to reflect predominantly on the quality of maternity care received for disabled women in England more recently.

\section{METHODS}

The main objective of this secondary analysis was to report on access to care and the quality of care received by disabled women who used the maternity services in 2015 in England, seeking a better understanding of the maternity care issues arising for women with different types of disability. In this paper, we:

- compare the perceptions and experiences of maternity care received by women with different types of disability and women with no disability;

- identify differences or gaps in care for disabled women which could be addressed.

\section{Study design and survey measure}

A structured cross-sectional study design was implemented by all National Health Service (NHS) trusts using a strict methodology and data collated by the Care Quality Commission (CQC) in $2015 .{ }^{14}$ The CQC is an independent regulator of health and social care in England and all NHS trusts providing maternity care and was responsible for the trust-based surveys using the same survey instrument. Modifications were made to the 2010 and then the 2013 CQC survey measures following consultation, focus groups and cognitive interviews which identified additional aspects of women's maternity care to be covered. While the survey continued to cover aspects of pregnancy, labour and birth, and postnatal care, more questions asked about women's access to care, communication with healthcare providers, involvement in decision making, awareness of birth choices and support for emotional well-being and physical health. Limited data on neonatal outcomes as well as sociodemographic characteristics including age, ethnicity, marital status and parity were also collected.

Postal surveys were sent to a minimum of 300 women from each trust who had given birth to a live baby in February 2015 (and possibly January 2015 for trusts with smaller numbers of births), 50945 women in total. Completing and returning the survey was considered as consent to take part in the study. Women who were younger than 16 years, those who had a stillbirth or whose baby died after birth, women who delivered in private settings and women without a UK postal address were excluded from the surveys. Up to two reminders were sent to non-respondents as required. A freephone language line provided translation services, and MENCAP also provided support to women with learning disabilities. ${ }^{14}$ The survey, reference $07 / \mathrm{MRE} 08 / 1$, was passed by the NRES Committee North West-Haydock in February 2015.

As in previous surveys, ${ }^{15}$ women were asked "Do you have any of the following long-standing conditions?" with seven options, including "No, I do not have a longstanding condition". Using the checklist, respondents were thus able to describe their disability and indicate if they had more than one disability. Five different disability groups were identified: physical (long-standing physical condition and long-standing illness), sensory (deafness or hearing impairment and blindness or partial sightedness), mental health problem, learning disability and multiple disabilities, that is, having two or more disabilities (see table 1).

\section{Statistical analysis}

The data presented are grouped in relation to access to care, the clinical care received and women's perceptions about the different phases of care. The categories used were those collected, and where variables were further aggregated for conciseness, this was based on clinical or policy relevance. The cut-offs are indicated in the tables. Univariate data analyses were carried out to compare the maternal characteristics and responses of disabled women to non-disabled women. $\chi^{2}$ statistics were used to compare study groups. Adjusted ORs and 95\% CI were weighted for variation in response rate by the trusts and adjusted for age, parity and ethnicity using binary logistic regression. Each of the subgroups, physical, sensory, mental, learning and multiple disability, was separately compared with the referent group of non-disabled women. Maternal characteristics and reports about care were compared with women who did not self-identify with any of the conditions listed above. The analyses were carried out in STATA V.13.

\section{RESULTS}

\section{Women's characteristics}

Overall, 20094 women completed and returned the survey, with a usable response rate of $41.2 \%$. Disabled women represented $9.5 \%$ (1958) of the total sample. 


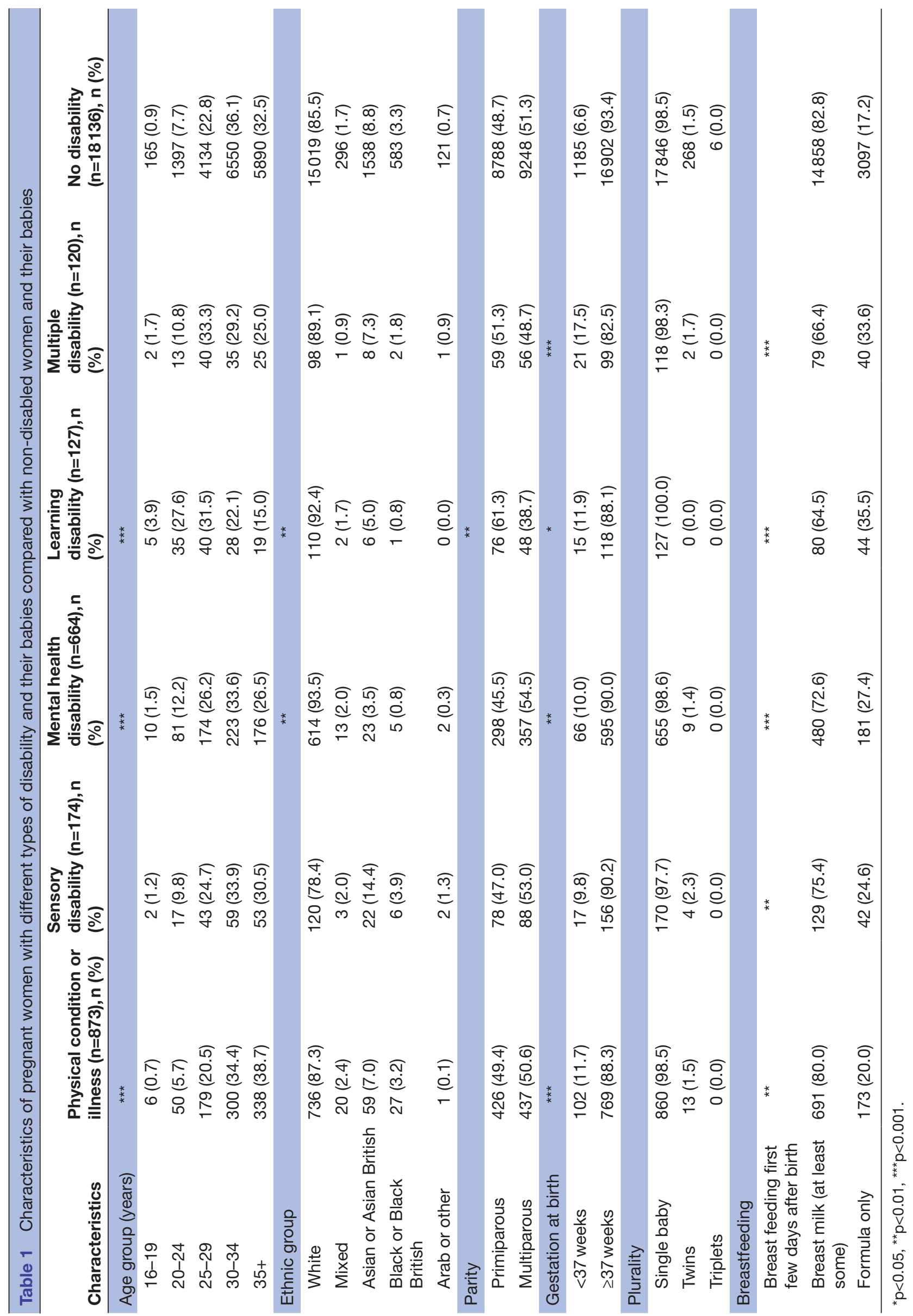


Compared with non-respondents, survey respondents were significantly more likely to be white, aged 30 years or more and primiparous ${ }^{14}$ which may affect the generalisability of results. Physical and mental health disabilities were most frequently identified. Of those with a disability, almost half reported having a physical disability (45\%), and a third of women identified with a mental health disability (34\%). Fewer women reported having a sensory disability $(8.7 \%)$, and small proportions of women reported having a learning disability $(6.5 \%)$ or more than one disability, most commonly a physical condition and mental health problem (6\%). More women with physical disability were 35 years and older than women with no disability ( $38.7 \%$ vs $32.5 \%$ ); however, women with mental health and learning disability were younger than women with no disability (table 1). White women were significantly more likely to report mental health and learning disabilities compared with all other ethnic groups. Similarly, primiparous women were significantly more likely than multiparous women to report learning disabilities. All disabled women were at a higher risk for delivering preterm compared with non-disabled women, particularly those with physical disability, mental health problems, learning disability and women with multiple disability (table 1). Across all groups, babies born to disabled were significantly less likely to be breast fed at the time of hospital discharge compared with non-disabled women.

\section{Access and care received}

Findings on access to maternity care and the care received are shown in table 2 .

Women with a physical disability accessed antenatal care similarly to those with no disability. However, those with a sensory disability were significantly less likely to see a health professional before 12 weeks' gestation and to have a later booking appointment (where a full history is taken and women are given their pregnancy notes) (table 2). There were no significant differences between the groups in continuity of care, with less than half of women in all groups seeing the same midwife for antenatal checks through the pregnancy. Choice in relation to place of birth differed for the disability groups: while only $9 \%$ of non-disabled women indicated that, for medical reasons, they had no choice about where they could have their baby, the comparable figure for women with a physical disability was $32 \%$ and for the other groups between $14 \%$ and $27 \%$. Clinical care differed across the groups in relation to labour and birth, with women with a physical condition significantly more likely to have intervention in the form of assisted vaginal births and planned or emergency caesarean section. Shortly after the birth, women with physical disability were slightly less likely to have skinto-skin contact with their baby, although nearly nine out of ten women did so.

While approximately a quarter of non-disabled women $(26 \%)$ stayed in hospital for more than 2 days after giving birth, more women in all the different types of disability groups did so, significantly more for women with physical, mental health, learning or multiple disabilities which may relate partly to method of delivery. Nearly half of the women with multiple disabilities $(45 \%)$ stayed longer than 2 days. More than $90 \%$ of women with and without disability received at least one postnatal home visit from a midwife, although this was slightly fewer for the physically disabled women. However, women with mental health or learning disability were significantly more likely to have received a home visit or seen a midwife in a clinic five or more times in the postnatal period. Women with physical or mental disability were less likely to report that advice about infant feeding was always available at evenings and weekends.

\section{Perceptions of care}

Women's views about the care received varied across the different groups (tables 3-5).

During pregnancy, women with physical disability, those with mental health conditions and women with more than one disability were all significantly less likely to feel that there was always time to ask questions at their appointments, to feel listened to, spoken to in a way they could understand, involved enough in decisions about their care, and if they had contacted a midwife, that they had been given the help they needed (table 3). All disabled women were significantly more likely to report negative experiences of pregnancy care, particularly in relation to always being spoken to by health professionals in a way that they could understand and, except for women with sensory loss, being involved in decisions about their care.

Perceptions of labour and birth care also differed between the groups (table 4 ). While $85 \%$ of non-disabled women reported that all staff who treated and examined them introduced themselves, significantly fewer women with physical disabilities and mental health conditions reported this $(76 \%$ and $74 \%$, respectively) (table 4$)$. Significantly fewer women in with physical, mental health and learning disabilities were likely to report definitely having confidence and trust in staff, fewer women in all disability groups reported always being spoken to so they could understand, and fewer women with physical, sensory and mental health disabilities reported that they were always treated with respect at this time. Significantly fewer women with physical disabilities $(65 \%)$ and mental health conditions $(65 \%)$ reported that they were always involved in decisions about their care compared with $76 \%$ of those with no disability. Similarly, while $83 \%$ of non-disabled women felt that their concerns during labour and birth were taken seriously, significantly fewer women with mental health problems or learning disability perceived this to be the case ( $74 \%$ and $72 \%$, respectively).

Women were asked whether they and their partner were left alone at a time when it worried them during labour or shortly after the birth and whether they received attention and help from a member of staff within a reasonable time. Feeling left alone and worried at some time was reported by a quarter of non-disabled women or with physical disability (25\% and 27\%, respectively) 


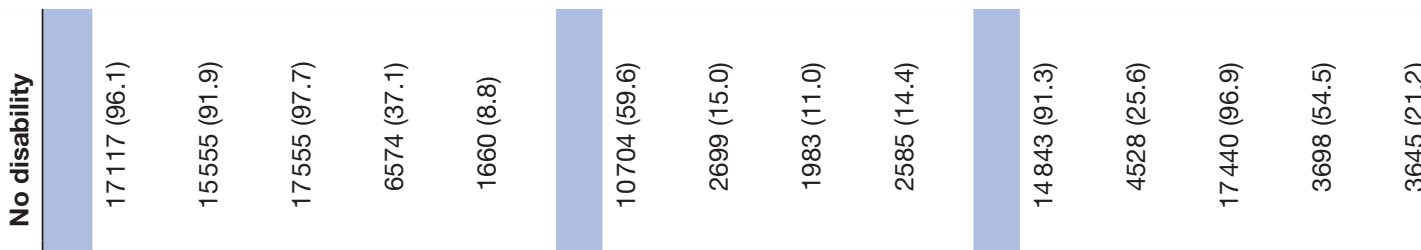

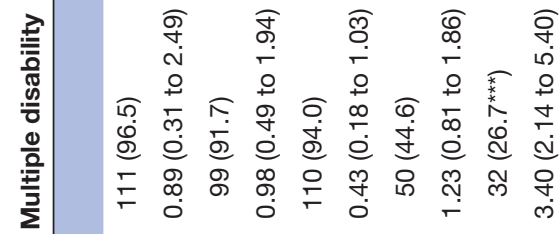

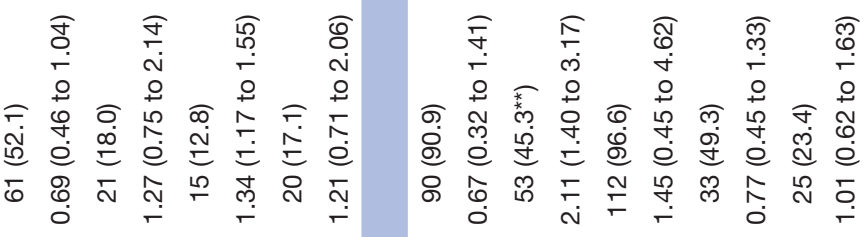

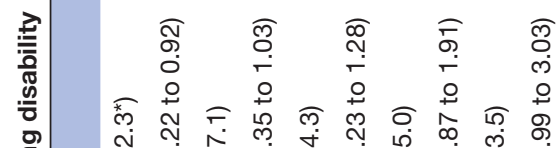

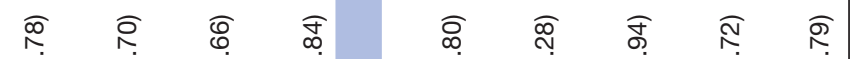
峞

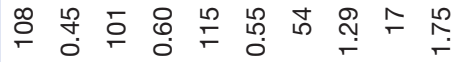

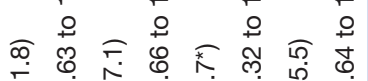

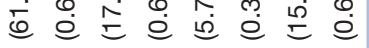

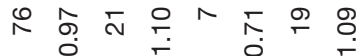

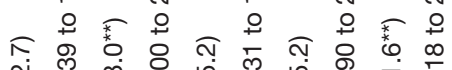

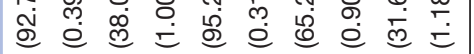

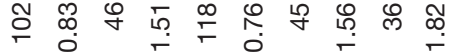

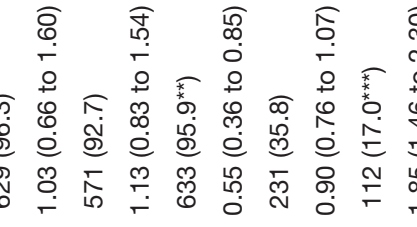

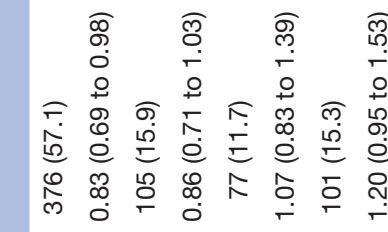

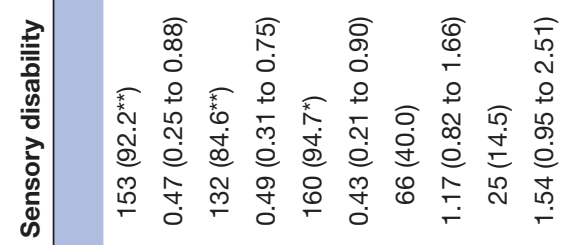

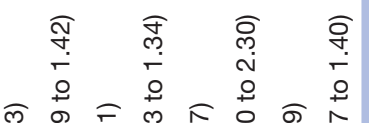

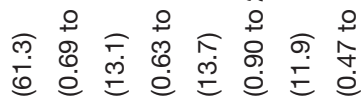

员

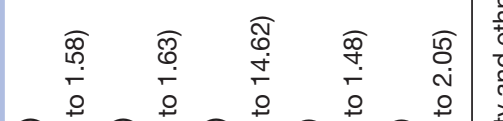

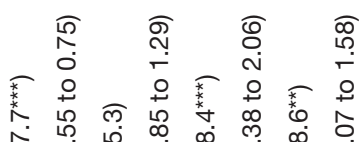

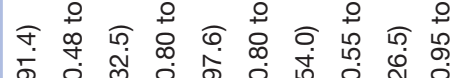

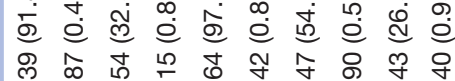
莳蛋 


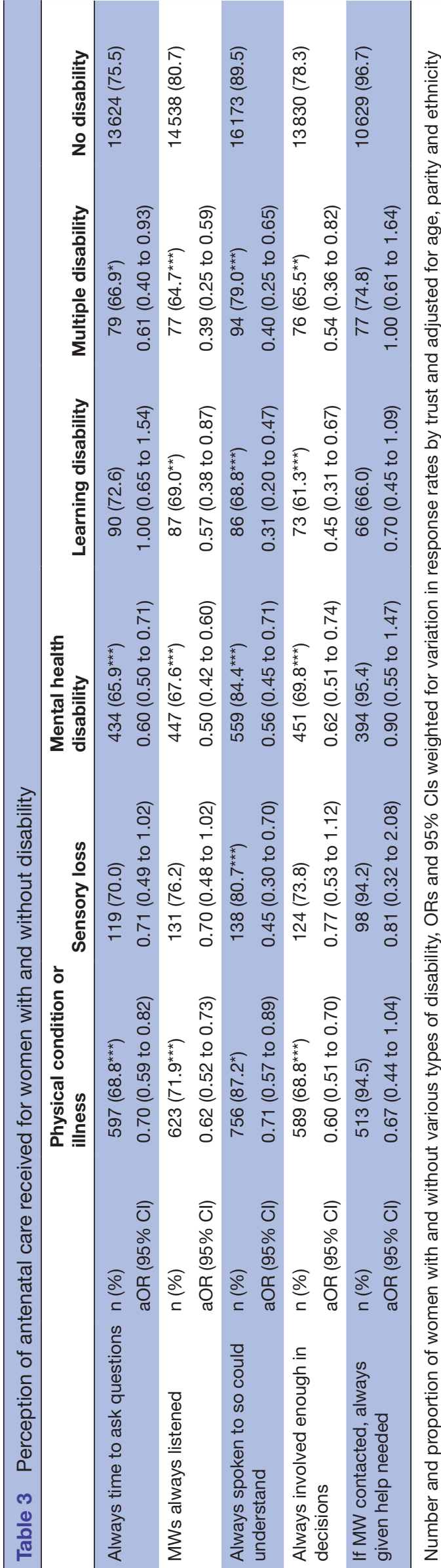

but significantly more so by the other disability groups. However, receiving attention within a reasonable time was reported by $65 \%$ of non-disabled women but significantly more so by women with a mental health condition $(69 \%)$ or a physical disability $(71 \%)$.

Perceptions of hospital and community postnatal care varied, with women who had a physical or mental health disability less likely to report a positive experience in both contexts (table 5). In hospital, they were significantly less likely to report always being treated with kindness and understanding or that their companion or partner was able to stay with them as much as they wanted. Once home, a third of those with a sensory disability would have liked to have seen midwives more often $(34 \%)$ as would women with learning disability $(30 \%)$, compared with a fifth $(20 \%)$ of non-disabled women. Over $70 \%$ of non-disabled women always felt listened to, definitely had confidence and trust in the midwives providing postnatal care at this stage, and, if a midwife was contacted, felt that they always received the help needed. However, for most variables, women with all forms of disability, especially mental health and learning disability, were significantly less likely to report so positively on these points.

Similarly, regarding infant feeding, women with physical or mental health disability were significantly less likely to report receiving active support and encouragement during the postnatal stay or, in the 6 weeks after the birth, to receive help and advice with feeding and the baby's health and progress.

\section{Checks and information on women's health and emotional well-being}

In the antenatal period, less than half of non-disabled women $(49 \%)$ reported that during their antenatal checks, midwives always appeared to be aware of their medical history (table 6). This was significantly even less likely for women with a physical or mental health disability (both 44\%). Among the midwives providing postnatal care, awareness was greater than for antenatal care for all groups. However, as with antenatal care, significantly fewer of those women with a physical or mental health disability felt that midwives were always aware of their medical history. Women were also asked if they had been given enough information about their physical recovery after the birth. Just over only half of those without disability reported that they had definitely been given this information (56\%). Some disability groups reported lower frequencies than this: women with a physical disability a mental health condition and multiple disability $(48 \%, 48 \%$ and $49 \%$, respectively) were all significantly less likely to have been given this information. Advice about contraception was less available to all disabled women, significantly so among those with a physical, mental health or learning disability.

Women with disability were more concerned that their personal circumstances had not been taken into account (65\% vs $74 \%)$. Women with mental health, learning or 


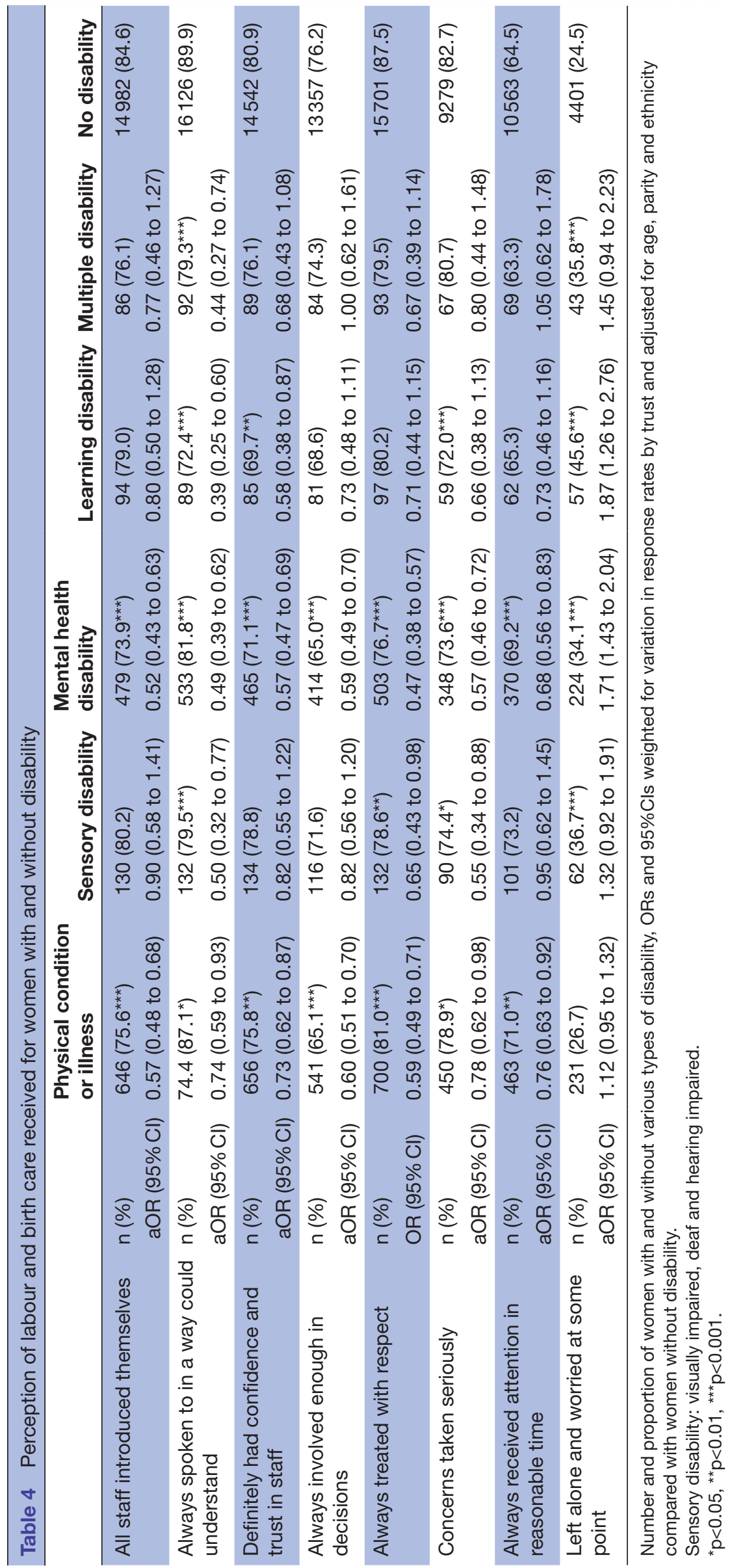




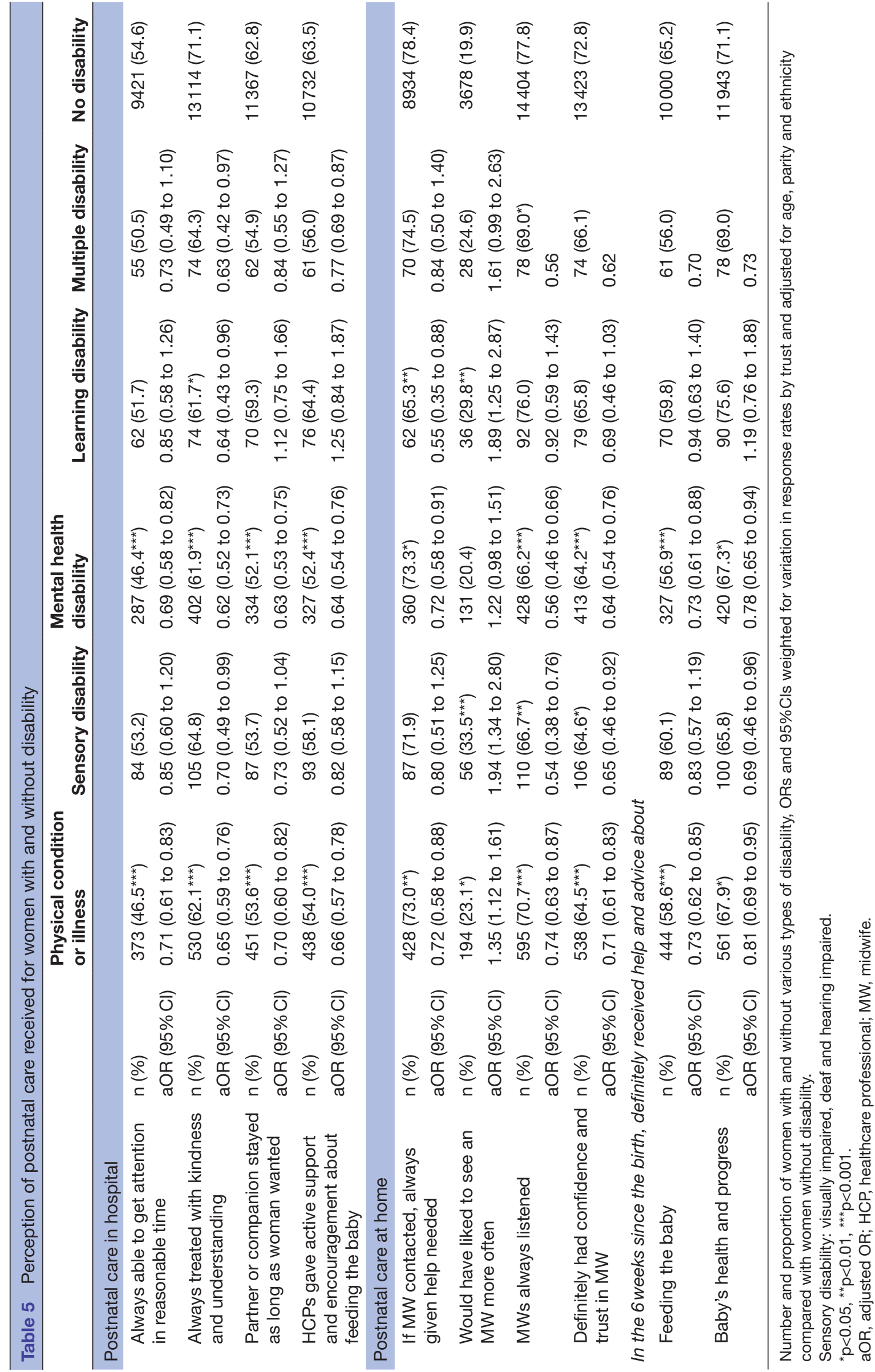




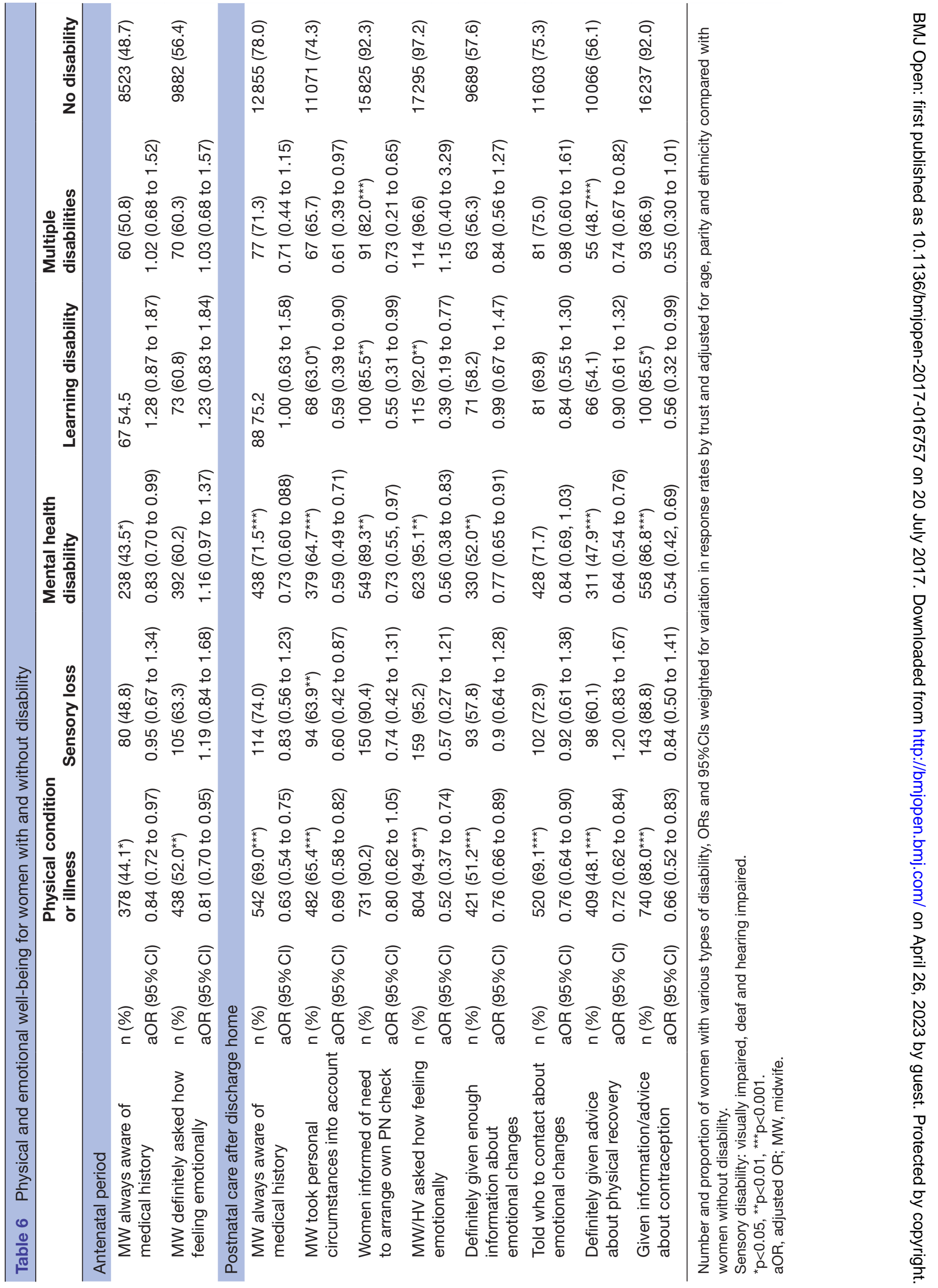


multiple disability were less likely to report being informed of the need to arrange their own postnatal check-up.

All women should be asked about their emotional wellbeing during pregnancy and postnatally. ${ }^{16}$ While just over half of those with no disability reported being asked about their emotional well-being during pregnancy $(56 \%)$, this was even less likely for those with a physical disability (52\%). In contrast, over $90 \%$ of women in all groups reported being asked about their emotional well-being postnatally, although some groups, especially women with a physical, mental health or learning disability, were still less likely to report having been asked. Women were also asked about being given information about the emotional changes that might be experienced after the birth. Fewer women overall (less than 60\%) reported being given enough information about possible changes in mood, and this was even less likely for women with physical disability and those with mental health problems $(51 \%$ and $52 \%$, respectively). Of non-disabled women, $75 \%$ were told who to contact for advice about any emotional changes, but only $69 \%$ of women with a physical disability reported this.

\section{DISCUSSION}

This study provides further evidence that disabled women have a poorer perception of care during pregnancy, childbirth and in the postnatal period which need to be recognised. The conditions giving rise to disability are extremely diverse, and some women may need more clinical or supportive care than others. Yet, such women often encounter negative attitudes toward their pregnancy. ${ }^{17} 18$ Disabled women are usually classified during their pregnancy as 'high risk', ${ }^{2}$ requiring more antenatal visits and more scans, as found in other studies. ${ }^{19}$ Arranging these intensive appointments can be difficult for some disabled women. There is a need for more specific services and more guidance and training for healthcare professionals caring for women with any disability during pregnancy.

This study shows that, in England in 2015, while care was more responsive in some respects for disabled women, such as more home visits after hospital discharge, disabled women overall perceived their care in more negative terms than non-disabled women. In particular, they felt that they were not always spoken to so that they could understand, listened to, did not always have time to ask questions, were not always sufficiently involved in decisions about their care, treated with respect, or their concerns taken seriously. Women with sensory, mental health, learning or multiple disabilities were more likely to be left alone at a time when it worried them during labour or shortly after birth. It may be that these women needed more reassurance and support or had more reason to be worried, but their concerns were not addressed by staff. It is also possible that disabled women who would, in general, have had more experience of the health service than non-disabled women were expressing their disillusionment with healthcare generally.
Communication barriers, deficits in health information and a lack of knowledge and awareness among healthcare professionals have been identified before ${ }^{20} 21$ and represent some of the attitudinal barriers faced by disabled women. Information needs to be distributed in accessible formats. Disability awareness and training for healthcare professionals as well as allocation of additional care time and flexible postnatal visiting could have a positive influence on care. In addition, the focus should be on women's abilities rather than their disabilities. Previous research has indicated that, while some staff were excellent, others provided 'unhelpful help', taking over, leading to feelings of disempowerment. ${ }^{22}$ Support through the transition from pregnancy to motherhood should also be considered by healthcare providers. ${ }^{5}$ Integrated care between different services, such as mental health and obstetric services, may be required to meet the needs of these groups.

These data from this survey highlight particular areas where maternity services need to improve to provide equal services to women with different types of disability. The greater number of questions in the 2015 survey focusing on specific aspects of maternity care contributes to a broader and more detailed picture of the care experienced by disabled women compared with previous surveys. Flexible and responsive services are needed by women with different types of disability. Specifically, women with physical disability are likely to need rather different personalised care and support from women with mental health disability. For example, women with physical conditions may need help with physical access, whereas those with mental health problems may need more emotional support than others. As we also concluded from our earlier study, empowering women and supporting their involvement in the decision-making process during pregnancy is a key area for improvement. ${ }^{13}$ Supported decision making may be necessary to enable some individuals to communicate their needs and choices. Individual women differ, and those with disability should be offered the same antenatal options, choices of birth place and pain relief as non-disabled women, unless their medical conditions contradict these options. Information should be accessible and in a comprehensive format. An early assessment of the maternity care required is crucial to forming a care plan with the women involved. Healthcare professionals need to plan ahead on how to meet the individual needs with the women themselves and to keep the conversation open and ongoing over the pregnancy and afterwards.

The needs of disabled women are still not fully met in the maternity services in England as evidenced here, and there is a clear need to document and assess the needs of this group. Research from Korea involving 410 physically disabled women points to high rates of abortion, miscarriage, caesarean section and low usage of contraception. ${ }^{23}$ In Switzerland, there are few guidelines and little regular assessment for women with psychiatric problems in the perinatal period. ${ }^{24}$ In qualitative studies in the USA and 
Canada, women with physical impairments reported numerous barriers to reproductive health services. ${ }^{17} 2526$ However, an Australian study illustrated the positive care experience possible for women attending a specialised childbirth and mental health antenatal clinic. ${ }^{27}$ The WHO global disability action plan 2014-2021 requires member states to strengthen the collection of relevant and internationally comparable data on disability and support research on disability and related services. ${ }^{3}$

\section{Strengths and limitations}

Strengths of this study include the fact that all the organisations providing maternity care in England participated and substantial numbers of women with different types of disability responded. Moreover, we report on women's own perspective on their care. All data in this survey were self-reported and collected retrospectively at 3 months postpartum. This may call into question the validity of the responses recalled from pregnancy. However, research into the accuracy of recall suggests that it is good. ${ }^{28-30}$ The survey response rate was low $(41 \%)$ which may affect the generalisability of the findings; however, weighting for non-response was used. Also, many possible associations were tested, and some significant associations may have arisen by chance. However, the high level of statistical significance of many of the associations reported mitigates against this. Analyses were limited to the data collected by CQC. Unfortunately, data were not collected on level of education, marital status, income level or urban/rural setting.

\section{Conclusion and implications for research and practice}

This study presents the findings of a 2015 maternity survey in England as they relate to disability. Using recently collected data, the study objectives were to investigate access to maternity care and the quality of that care as reflected in women's perceptions, exploring differences in the experience of women with different types of disability.

Disabled women perceived greater problems regarding their maternity care, communication and involvement in decision making than non-disabled women. Those with a physical disability or long-standing illness perceived problems regarding inadequate or inappropriate communication, limited involvement in decision making, and being able to establish a trusted and respected relationship with clinical staff are areas for improvement for women in this group. For women with sensory disability, having information delivered in an appropriate format was particularly important. It may be helpful for staff caring for these women to allow more time to communicate effectively throughout their maternity care.

In order to provide more appropriate care for women with a mental health disability, a longer hospital stay and more frequent midwife visits may be required. In this group, many aspects of maternity care were not perceived as positively as for other groups, particularly they felt that they were not always listened to, did not have time to ask questions, were not sufficiently involved in decisions about their care, treated with respect or had their concerns taken seriously.

Similarly for women with multiple disabilities, improvements in communication and involvement in decision making are needed. For women with a learning disability, aspects of care concerning communication and involvement in decisions, feeling listened to and supported, particularly during labour and birth, were highlighted as lacking, and specific efforts are needed to improve the quality of care experienced.

Further research could focus on specific groups and involve qualitative and quantitative methods. Studies of attitudes and knowledge of healthcare providers, including the way in which stereotypes may operate, would also be useful in understanding the differences in care and disabled women's perceptions described.

Healthcare professionals sometimes lack sufficient awareness and experience to respond effectively to the needs of disabled women during pregnancy and the early postnatal period. As reported elsewhere, ${ }^{22}$ disabled women want to be assisted to do things themselves, rather than having things done for them. To achieve satisfactory maternity care for all women, the needs and voices of women with disabilities should not only be referred to in the strategy and policy documents of healthcare providers but also embodied in their provision and practice, allowing more time for appointments and additional support staff and equipment as required.

Acknowledgements This work reports on an independent study which was funded by the Policy Research Programme in the Department of Health (DH) in the United Kingdom. The views expressed are not necessarily those of the DH. The Care Quality Commission was responsible for the original survey and granted access to the data. We are most grateful to women who responded to the survey.

Contributors All three listed authors have contributed to this paper: MR designed the analysis plan, $\mathrm{JH}$ and RM carried out the analyses, RM drafted the main manuscript and all authors contributed to the interpretation of the findings and refining the manuscript. All three authors read and approved the final version of the manuscript.

Funding This study was funded by the Policy Research Programme in the Department of Health (DH) in the UK.

Competing interests None declared.

Provenance and peer review Not commissioned; externally peer reviewed.

Data sharing statement The women's trust-based data used in this study were accessed from CQC that was responsible for the survey.

Open Access This is an Open Access article distributed in accordance with the Creative Commons Attribution Non Commercial (CC BY-NC 4.0) license, which permits others to distribute, remix, adapt, build upon this work non-commercially, and license their derivative works on different terms, provided the original work is properly cited and the use is non-commercial. See: http://creativecommons.org/ licenses/by-nc/4.0/

(C) Article author(s) (or their employer(s) unless otherwise stated in the text of the article) 2017. All rights reserved. No commercial use is permitted unless otherwise expressly granted.

\section{REFERENCES}

1. Blackford KA, Richardson H, Grieve S. Prenatal education for mothers with disabilities. J Adv Nurs 2000;32:898-904. 
2. Walsh-Gallagher D, Sinclair M, Mc Conkey R. The ambiguity of disabled women's experiences of pregnancy, childbirth and motherhood: a phenomenological understanding. Midwifery 2012;28:156-62.

3. World Health Organization. Disability and rehabilitation. 2016. http:// www.who.int/disabilities/data/en/ (11 Apr 2017).

4. Hague G, Thiara R, Mullender A. Disabled women, domestic violence and social care: the risk of isolation, vulnerability and neglect. $\mathrm{Br} J$ Soc Work 2011;41:148-65.

5. Lawler D, Lalor J, Begley C. Access to maternity services for women with a physical disability: a systematic review of the literature. Int $J$ Childbirth 2013;3:203-17.

6. Thomas C. Medicine, gender, and disability: disabled women's health care encounters. Health Care Women Int 2001;22:245-62.

7. Thomas C. Disability: getting it "right". J Med Ethics 2008;34:15-17.

8. Lipson JG, Rogers JG. Pregnancy, birth, and disability: women's health care experiences. Health Care Women Int 2000;21:11-26.

9. Clark L. Accessible health information: Liverpool Central Primary Care Trust Project Report Liverpool. 2002. http:// pf7d7vi404s1dxh27mla5569.wpengine.netdna-cdn.com/files/library/ Clark-Laurence-liverpool-NHS.pdf (11 Apr 2017).

10. Steinberg EJ, 2006. Deaf mothers and reproductive healthcare: identifying inequalities and documenting experiences. Presented at the Wellington Park Hotel, Belfast Conference on Equality and Social Inclusion in the 21st Century; 1 February, 2006.

11. O'Hearn A. Deaf women's experiences and satisfaction with prenatal care: a comparative study. Fam Med 2006;38:712-6.

12. Jesse DE, Dolbier CL, Blanchard A. Barriers to seeking help and treatment suggestions for prenatal depressive symptoms: focus groups with rural low-income women. Issues Ment Health Nurs 2008;29:3-19.

13. Redshaw M, Malouf $\mathrm{R}$, Gao $\mathrm{H}$, et al. Women with disability: the experience of maternity care during pregnancy, labour and birth and the postnatal period. BMC Pregnancy Childbirth 2013:13:174.

14. Care Quality Commission. 2015: Maternity Survey: quality and Methodology. London, 2016.

15. Care Quality Commission. National findings from the 2013 survey of women's experiences of maternity care. London: CQC, 2013.

16. National Collaborating Centre for Mental Health. Antenatal and postnatal mental health. clinical management and service guidance. London: National Institute for Health and Care Excellence, 2014.
17. Becker H, Stuifbergen A, Tinkle M. Reproductive health care experiences of women with physical disabilities: a qualitative study. Arch Phys Med Rehabil 1997;78:S26-S33.

18. Gill C, Kerotoski MA, Turk NMA. Becoming visible: personal health experiences of women with disabilities. DM women with physical disabilities: achieving and maintaining health and wellbeing: Baltimore: Pall H. Brookes, 1996:5-15. p.

19. Mitra M, Clements KM, Zhang J, et al. Maternal characteristics, pregnancy complications, and adverse birth outcomes among women with disabilities. Med Care 2015;53:1027-32.

20. Smeltzer SC, Sharts-Hopko NC, Ott BB, et al. Perspectives of women with disabilities on reaching those who are hard to reach. $J$ Neurosci Nurs 2007;39:163-71.

21. Prilleltensky O. A ramp to motherhood: the experiences of mothers with physical disabilities. Sex Disabil 2003;21:21-47.

22. Thomas C, Curtis P. Having a baby: some disabled women's reproductive experiences. Midwifery 1997;13:202-9.

23. Lee O, E-k O, Oh $\mathrm{H}$. A wise wife and good mother: reproductive health and maternity among women with disability in South Korea. Sex Disabil 2005;23:121-44.

24. Amiel Castro RT, Schroeder K, Pinard C, et al. Perinatal mental health service provision in Switzerland and in the UK. Swiss Med Wkly 2015;145.

25. Blackford KA, Richardson H, Grieve S. Prenatal education for mothers with disabilities. J Adv Nurs 2000;32:898-904.

26. lezzoni LI, Wint AJ, Smeltzer SC, et al. Physical accessibility of routine prenatal care for women with mobility disability. $J$ Womens Health 2015;24:1006-12.

27. Hauck Y, Allen S, Ronchi F, et al. Pregnancy experiences of Western Australian women attending a specialist childbirth and mental illness antenatal clinic. Health Care Women Int 2013;34:380-94.

28. Bat-Erdene U, Metcalfe A, McDonald SW, et al. Validation of Canadian mothers' recall of events in labour and delivery with electronic health records. BMC Pregnancy Childbirth 2013;13(Suppl 1):S3

29. Tate AR, Dezateux C, Cole TJ, et al. Factors affecting a mother's recall of her baby's birth weight. Int J Epidemiol 2005;34:688-95.

30. Quigley MA, Hockley C, Davidson LL. Agreement between hospital records and maternal recall of mode of delivery: evidence from 12391 deliveries in the UK Millennium Cohort Study. BJOG 2007;114:195-200. 